\title{
Shear-thickening fluids in biologically relevant agents
}

\author{
Peter Kilbride $^{\mathrm{a}, *}$, Marina Vazquez Rull ${ }^{\mathrm{a}}$, Adam Townsend ${ }^{\mathrm{b}}$, Helen Wilson ${ }^{\mathrm{b}}$ and John Morris ${ }^{\mathrm{a}}$ \\ ${ }^{\mathrm{a}}$ Asymptote Ltd., General Electric Healthcare, Cambridge, UK \\ ${ }^{\mathrm{b}}$ Department of Mathematics, University College London, London, UK
}

Received 23 October 2018

Accepted 13 February 2019

\begin{abstract}
.
BACKGROUND: The rheology of shear thickening fluids is well characterized for many physical applications, however the literature surrounding biologically or cryobiologically compatible shear thickening fluids is less well understood.

OBJECTIVE: This study examined fluids consisting of corn-derived hydroxyethyl starch with a variety of sugars and cryoprotectants to characterize their shear-rate viscosity relationship. The objective was to establish if cryobiologically relevant materials could be used to afford biologics protection through shear-thickening.

RESULTS: Fluids consisting of 50\% hydroxyethyl starch by weight exhibited shear thickening with a variety of cryoprotectants. Lowering the temperature of the fluid both reduced critical shear rates and enhanced thickening magnitude. Starch derived from corn, wheat, and rice all exhibited non-Newtonian shear-dependent viscosity behaviour at $50 \%$ by weight in water. Between the starch sources however, the shear-rate viscosity relationship varied widely, with wheat-derived starch shear thinning, and the remaining starches forming shear thickening fluids. Different starch sources had different baseline viscosities, critical shear rates, and rates of viscosity increase.

CONCLUSIONS: This study established that shear thickening is compatible with cryobiologically relevant agents, particularly so at lower temperatures. This forms the basis for harnessing these phenomena in biological processes such as cryopreservation.
\end{abstract}

Keywords: Cryopreservation, shear-thickening, starch, colloids, temperature-dependence

\section{Introduction}

Shear thickening fluids have been widely studied for their unusual properties [1-5]. The majority of studies and uses of shear thickening materials are to be found in industrial applications with the literature surrounding biologically relevant applications more sparse. This is as opposed to shear thinning nonNewtonian fluids which have received considerable attention in biology, most notably studies of the shearthinning behaviour of blood [6,7]. Low temperature preservation of biological systems may benefit from the inclusion of non-Newtonian fluids.

Cryobanking, the long-term preservation of biological materials at low temperatures, achieves successful outcomes through one of two separate approaches. The first of these, known as traditional or slow cooling cryopreservation, involves cooling a sample in the presence of ice. While successful for a large number

\footnotetext{
*Corresponding author: Peter Kilbride, Asymptote Ltd., General Electric Healthcare, Sovereign House, Vision Park, Cambridge, CB24 9BZ, UK. E-mail: peter.kilbride@ge.com.
} 
of cell suspensions, application to larger structures (larger than a few hundred $\mu \mathrm{m}$ ) has proved difficult due to mechanical ice damage and stresses [8]. To overcome this, a second method of cryopreservation can be attempted known as vitrification. This process uses high concentrations of cryoprotectants (CPAs), combined with rapid cooling rates to prevent ice formation during cooling resulting in stable ice-free storage of the biologic below the glass transition temperature. Vitrification, which can be considered preservation in an extremely viscous liquid, has had notable successes and widespread application in areas such as embryo cryopreservation, however the high CPA concentrations required have proven unacceptably toxic and rapid cooling rates have proven impractical for biologics larger than approximately $1 \mathrm{~mm}^{3}$ [8-11], where prolonged exposure to the CPA is required to allow diffusion into the biologic interior, this is despite the pressing need for tissue and organ cryopreservation [8,12-14]. Control over the viscous conditions experienced during cryopreservation may aid the cooling process by allowing near-solidification on demand.

Several known shear thickening colloids are already used biologically. These represent an obvious starting point for examining potential candidates for use in non-Newtonian aided cryopreservation, as biological compatibility has already been established with these colloids. Perhaps the best known colloidal agent - corn starch - is the source of hydroxyethyl starch (HES), which has many clinical applications $[2,4,15]$. Nano-spheres are also increasingly used in areas of medicine such as cancer treatment or therapeutic delivery $[16,17]$.

This study examines the shear response of several biologically compatible agents to which HES has been added to allow shear-thickening. The agents are considered as suspensions in a binary system, as well as in more complicated systems containing sugars and dimethyl sulphoxide (DMSO) - sugars and DMSO are known cryoprotectants and their inclusion in cryopreservation medium is essential $[8,18]$. The impact of different starch sources is examined, before the impact of temperature on both the underlying viscosity and critical shear rates is established.

\section{Materials and methods}

Unless otherwise indicated, all reagents were sourced from Sigma-Aldrich (Gillingham, UK). All concentrations are given by $\%$ weight unless otherwise indicated.

\subsection{Viscometer measurements}

All viscosity measurements were carried out using an Anton Paar (St. Albans, UK), RheolabQC Rheometer with a DG42 double concentric cylinder attachment, which established viscosity using rotational rheometry. The system consists of two concentric cylinders, between which a mix under test is placed. Into this mix, a third cylinder is inserted and rotated at a certain rate to give a specific shear (each gap was $1.2 \mathrm{~mm}$ wide, and was consistent for all measurements), which is operable in the range $0-180^{\circ} \mathrm{C}$. In the present study temperatures as low as $-1^{\circ} \mathrm{C}$ gave accurate results when validating with a knownviscosity standard. To measure viscosity, $12 \mathrm{ml}$ of each sample fluid was separately prepared as per Section 2.3. After complete mixing, the fluid was added to the rheometer. The rheometer would then rotate through the range of shear rates of interest. Viscometric readings were recorded using Rheocompass software (Anton Paar, St. Albans, UK), which calculated viscosity based on shear rate and measured friction of rotation on the cylinder walls. All measurements were repeated three times sweeping up and sweeping down to ensure precision. Any data where the standard deviation was above $10 \%$ through experimental 
noise was repeated for an additional sweep to reduce uncertainty. No time-dependency was seen during experimental runs after complete mixture preparation (Section 2.3).

T-type thermocouples were used to record temperature. These were attached to a picologger unit (Picotechnology, St. Neots, UK), and temperatures recorded using picologger software (Picotechnology, St. Neots, UK). To the data, a line of best fit was added, the method used stated in the figure legends.

\subsection{Viscosity measurements at non-ambient temperatures}

Before cooling, T-type thermocouples were added to both the inner and outer wall of the viscometer measuring attachment. A variable temperature bath was prepared using a Neslab cold circulating system (Thermo Scientific, Waltham, MA, USA) with antifreeze (TRIPLE QX Blue Antifreeze) as a working fluid. The target temperature was confirmed by a thermocouple placed into the bath. A sample fluid was placed into the measuring attachment of the rheometer and lowered into the variable-temperature bath.

Temperature readings were taken as the cylinder's temperature equilibrated until the target temperature was reached. Rheometric readings were then carried out while ensuring no change in temperature occurred.

\subsection{Mixture preparation}

The following starches used HES (Sigma, H6382), starch from wheat (Sigma, S5127), and starch from rice (Sigma, S7260). All sample fluids were prepared fresh at room temperature $\left(22^{\circ} \mathrm{C}\right)$ and mixed thoroughly using a magnetic stirrer for at least 10 minutes, before use to ensure homogeneity. Samples were prepared fresh and used within 10 minutes of mixing to prevent separation of materials, and to ensure that there was no time-dependence with any of the tested materials, either at constant or varying shear. No hysteresis was seen over the scan period (10 seconds per measurement), or between separate measurements at the same shear rate.

\subsection{Microscopy}

Samples were prepared and placed between two microscope cover slips. Images were taken using an Olympus BX51 microscope at 10X optical magnification, with an attached CCD camera.

\section{Results}

\subsection{Shear-thickening with HES, Water, and DMSO}

The data in Fig. 1 established that mixes of the cornstarch-derived hydroxyethyl starch (HES, Sigma, H6382), exhibited non-Newtonian shear-thickening behaviour at room temperature. A binary system of $50 \%$ HES and $50 \%$ water (by weight) had a measured viscosity of $133.4 \pm 2.3 \mathrm{mPa} \cdot \mathrm{s}(n=5)$ below $12 \mathrm{~s}^{-1}$. This rose to above $915 \mathrm{mPa} \cdot \mathrm{s}$ above $77 \mathrm{~s}^{-1}$. Replacing some water with dimethyl sulphoxide leaving 50\% HES, 40\% water and 10\% dimethyl sulphoxide (DMSO, Sigma, D4540) did not remove the non-Newtonian behaviour, although the thickening behaviour was observed over a wider range of shear rates as can be seen in Fig. 1. Overall, viscosity was measured lower at $66.1 \pm 1.7 \mathrm{mPa} \cdot \mathrm{s}(n=5)$ below $2 \mathrm{~s}^{-1}$, this increased to above $355 \mathrm{mPa} \cdot \mathrm{s}$ above $66 \mathrm{~s}^{-1}$. 


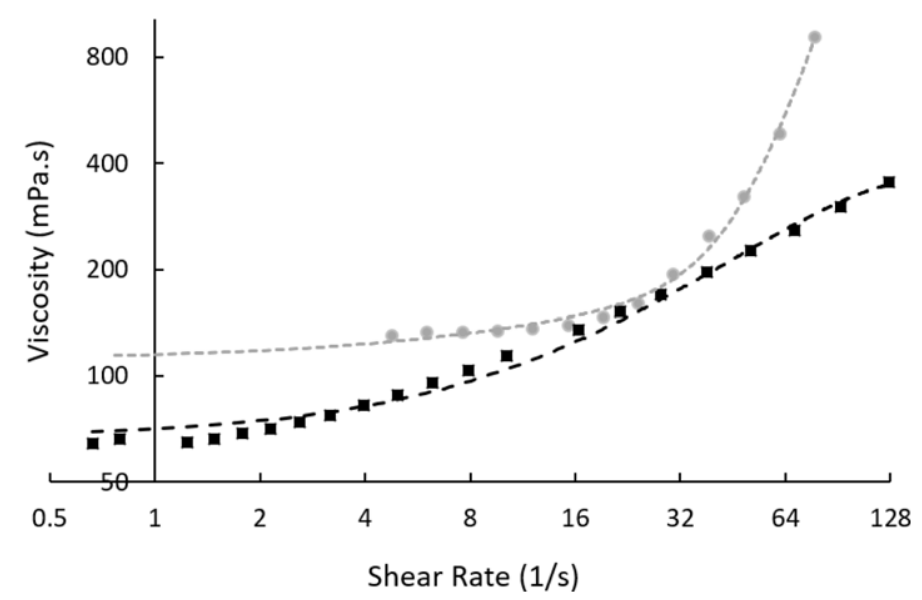

Fig. 1. Viscosity and shear relation of a 50\% hydroxyethyl starch and 50\% water binary system (grey circles, with exponential fitting line) and a 50\% hydroxyethyl starch, $40 \%$ water, and 10\% dimethyl sulphoxide tertiary system (black squares, with secondorder polynomial fitting line). Strong shear thickening was observed in the binary system around $30 \mathrm{~s}^{-1}$ as measured by rotational techniques. Shear-thickening was lesser in the tertiary system with a wider critical shear range. Measurements were taken at $22^{\circ} \mathrm{C}$.

\subsection{Shear-thickening with HES, water, sugars and glycerol}

Fluids consisting of 50\% HES, 40\% water, and 10\% of either glucose (Sigma, G8270), glycerol (Sigma, G9012), or sucrose (Sigma, S0389) all exhibited shear-thickening behaviour at $22^{\circ} \mathrm{C}$, as can be seen in Fig. 2. Both solutions with glucose or glycerol had the onset of shear thickening at approximately $2 \mathrm{~s}^{-1}$, with viscosities of $92.1 \pm 2.2 \mathrm{mPa} \cdot \mathrm{s}(n=7)$ and $106.5 \pm 1.3 \mathrm{mPa} \cdot \mathrm{s}(n=8)$ measured respectively. Solutions with sucrose had a stable viscosity below approximately $10 \mathrm{~s}^{-1}$ of $287.0 \pm 6.1 \mathrm{mPa} \cdot \mathrm{s}(n=10)$. Viscosities were measured to rise to above $776 \mathrm{mPa} \cdot \mathrm{s}$ at $154 \mathrm{~s}^{-1}, 662 \mathrm{mPa} \cdot \mathrm{s}$ at $167 \mathrm{~s}^{-1}$, and $1495 \mathrm{mPa}$ $\cdot \mathrm{s}$ at $54 \mathrm{~s}^{-1}$ for glucose, glycerol, and sucrose mixtures respectively. Tests were also conducted on the solution without starches. $10 \%$ DMSO in water had a viscosity of $1.14 \pm 0.3 \mathrm{mPa} \cdot \mathrm{s}(n=10)$, and $10 \%$ glycerol in water had a viscosity of $1.22 \pm 0.4 \mathrm{mPa} \cdot \mathrm{s}(n=10)$, both at $22^{\circ} \mathrm{C}$. Various concentrations of sugars have been characterised over a range of concentrations and temperatures [19]. Solutions without starch displayed Newtonian behaviour.

\subsection{Impact of temperature reduction on shear thickening of biological reagents}

The impact of reducing temperature from $22^{\circ} \mathrm{C}$ to $0^{\circ} \mathrm{C}$ is shown in Fig. 3. Mixtures of $50 \%$ HES, $40 \%$ water, and $10 \%$ of either glucose (panel A), raffinose (panel B, Sigma, R0514), sucrose (panel C), DMSO (panel D), fructose (panel E, Sigma, F0127), or lactose (panel F, lactose, L3625) were examined. In all cases (except lactose where thickening occurred over a range too narrow to accurately measure), a reduction in temperature resulted in a reduction in critical shear rates. The intensity of shear thickening was also greater in cooler samples $(p<0.05)$. The relative rate of increase in viscosity is shown for all tested samples in Fig. 6. 


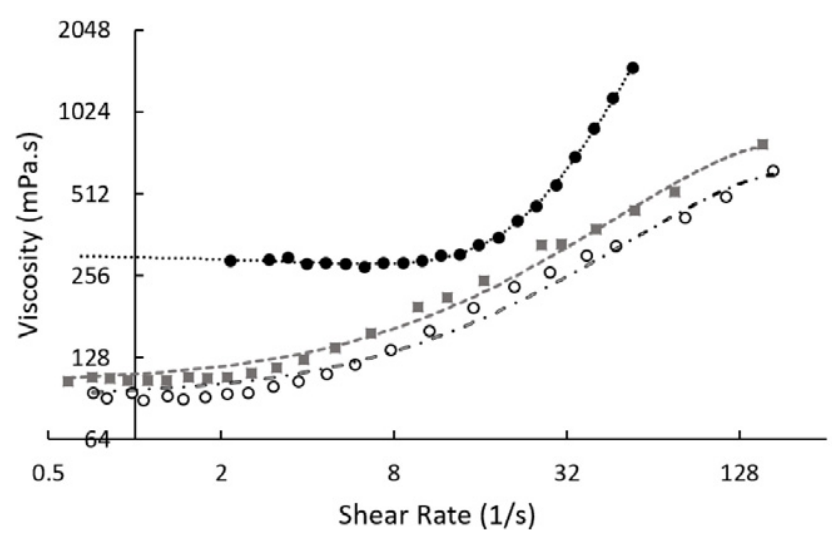

Fig. 2. The shear-thickening behaviour observed with a $50 \%$ hydroxyethyl starch, $40 \%$ water, and $10 \%$ cryoprotectant mix. Thickening was observed in all systems using rotational techniques where the cryoprotectant sucrose (black circles, with exponential fitting line), glycerol (grey squares, with second-order polynomial fitting line), and glucose (black rings, with secondorder polynomial fitting line) were present in the mix. Measurements were taken at $22^{\circ} \mathrm{C}$.

\subsection{Impact of temperature in a quaternary system}

Figure 4 shows that at increasingly lower temperatures, the rate at which a mixture of 50\% HES, 35\% water, $10 \%$ sucrose, and 5\% DMSO started to show shear-dependent viscosity was $0.05 \mathrm{~s}^{-1}$ at $-1^{\circ} \mathrm{C}$, rising to $30 \mathrm{~s}^{-1}$ at $50^{\circ} \mathrm{C}$. The relative magnitude of shear thickening was also observed to be greater at lower temperatures, with a 2 orders of magnitude increase in viscosity occurring over only a $10 \%$ increase in shear rate at $-1{ }^{\circ} \mathrm{C}$. The equilibrium freezing point of this solution is approximately $-3^{\circ} \mathrm{C}$.

\subsection{Impact of different starch sources}

The source of starch is extremely important in dictating shear-dependent viscosity. All three tested sources - wheat (Sigma, S5127), corn starch derived HES, and rice (Sigma, S7260), exhibited nonNewtonian effects at $50 \%$ weight in water at $22^{\circ} \mathrm{C}$. These data are plotted in Fig. 5.

Starch derived from rice exhibited both shear-thickening and thinning behaviour. Between 0.1 and $0.2 \mathrm{~s}^{-1}$ viscosity was measured at $7800 \pm 400 \mathrm{mPa} \cdot \mathrm{s}(n=4)$. This fell to $2147 \pm 47 \mathrm{mPa} \cdot \mathrm{s}(n=6)$ between 5 and $15 \mathrm{~s}^{-1}$. Further increases in shear rate resulted in increasing viscosity.

A mixture consisting of $50 \%$ wheat derived starch had the lowest viscosity and exhibited shearthickening behaviour. Viscosity was recorded at $24.8 \pm 1.3 \mathrm{mPa} \cdot \mathrm{s}(n=5)$ between 20 and $60 \mathrm{~s}^{-1}$. This rose above $150 \mathrm{mPa} \cdot \mathrm{s}$ at shear rates greater than $70 \mathrm{~s}^{-1}$. Data from HES is shown in Fig. 1.

Microscopy of the 3 different sources was taken and is shown in Fig. 7. Starch derived from wheat was found to have an average diameter of between 10-20 $\mu \mathrm{m}$, HES had an average diameter of $10 \mu \mathrm{m}$, and starch derived from rice had an average diameter $<5 \mu \mathrm{m}$.

\section{Discussion}

This study aimed to provide an experimental basis for shear-thickening behaviour in systems compatible with biological materials, and specifically those with a cryobiological application. HES is a non-ionic derived starch, most commonly sourced from corn starch. While corn starch mixtures are well known 

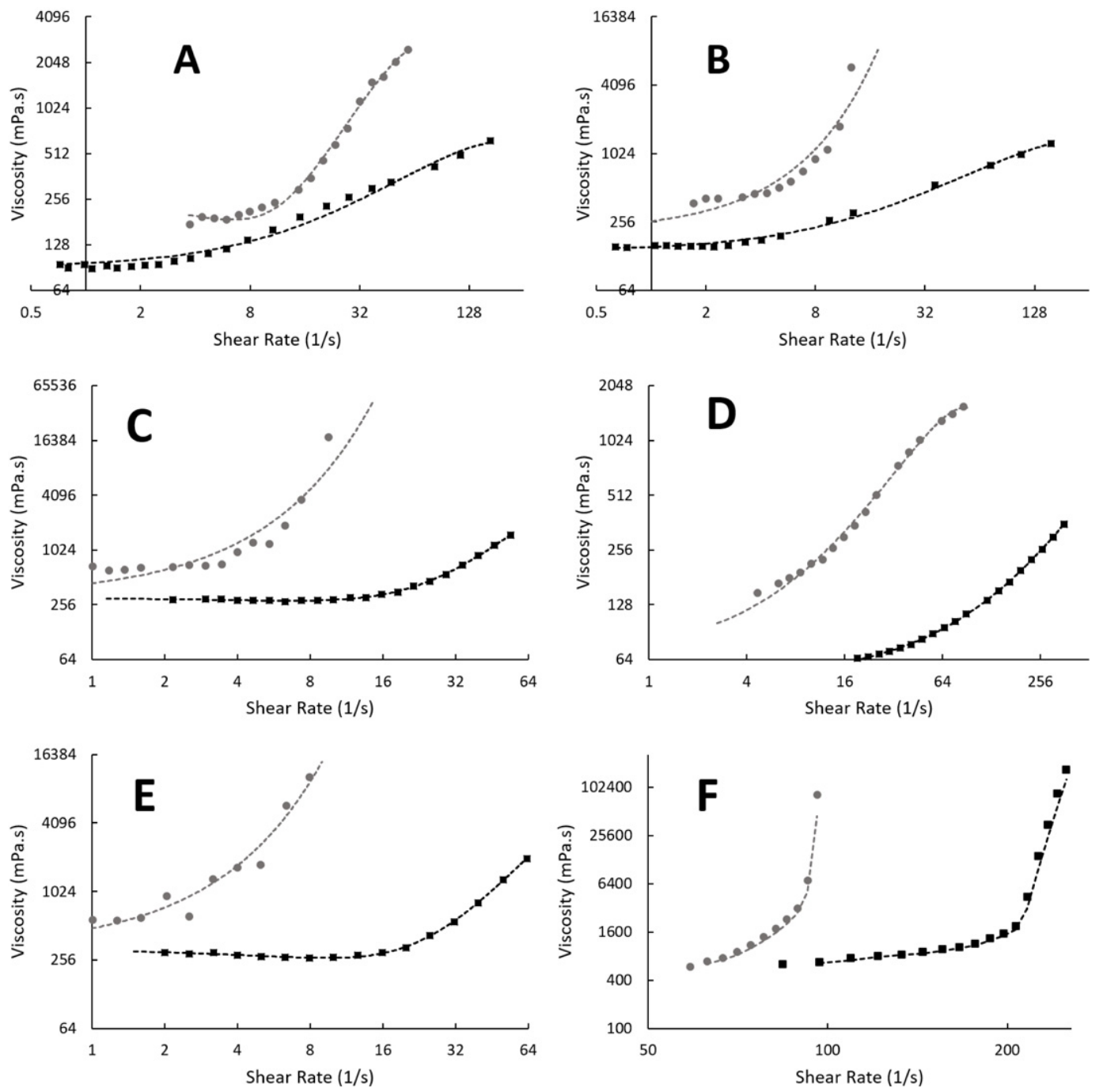

Fig. 3. The impact of lowering temperature on the critical shear rates and magnitude of shear-thickening effects on a range of hydroxyethyl starch and water-based mixtures. The mixtures contained a $50 \%$ hydroxyethyl starch, $40 \%$ water, and $10 \%$ cryoprotectant or sugar. The examined cryoprotectant or reagent was (A) glucose, (B) - raffinose, (C) sucrose, (D) dimethyl sulphoxide, (E) fructose, and (F) lactose. All measurements were examined using rotational techniques with readings taken at $22 \mathrm{C}$ (black squares), and $0^{\circ} \mathrm{C}$ (grey circles). Thickening was observed to occur at lower shear rates at lower temperatures in all examined mixtures. For readings taken at $22^{\circ} \mathrm{C}$, lines were fitted using 2 nd order polynomial. For those at $0^{\circ} \mathrm{C}$, exponentials were used, except in panes A and D where 3 rd order polynomials were employed.

to exhibit shear-thickening behaviours $[2,4,15]$, this study established that the processing involved to allow their biological use (conversion to HES) does not remove this ability. HES has several clinical applications [6,20-22], and so cytotoxicity issues would not be expected.

The addition of other reagents essential for cryoprotection at low temperatures - such as monosaccharides, disaccharides, trisaccharides, glycerol, or DMSO - does not prevent the shear-thickening behaviour. DMSO was seen to reduce the viscosity of the system, while sugars were seen to increase the viscosity. Between the sugars, the impact of $10 \%$ inclusion on viscosity was not consistent, for example sucrose 


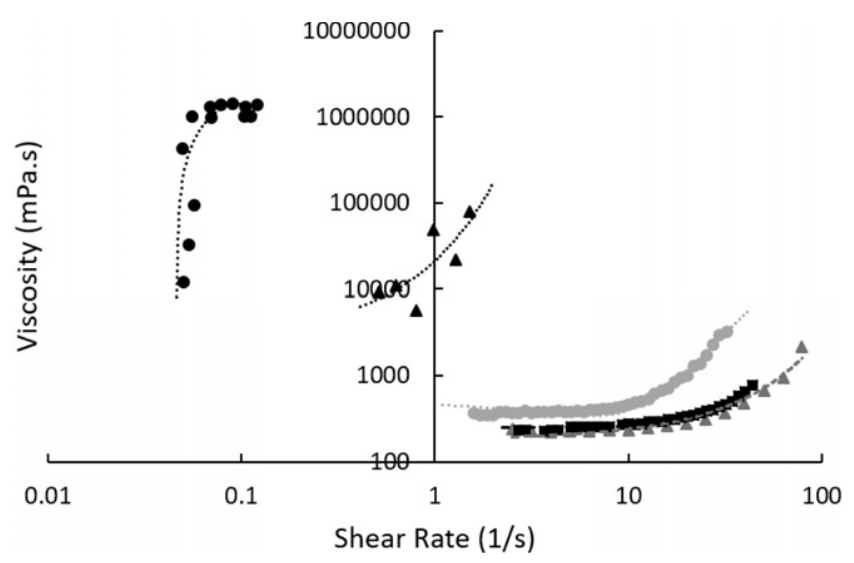

Fig. 4. The impact of temperature changes on the shear dependent viscosity of a $50 \%$ hydroxyethyl starch, $35 \%$ water, $10 \%$ sucrose, and 5\% dimethyl sulphoxide system. All measurements were examined using rotational techniques with readings taken at $-1^{\circ} \mathrm{C}$ (black circles, with second-order polynomial fitting line), $10^{\circ} \mathrm{C}$ (black triangles, with exponential fitting line), $30^{\circ} \mathrm{C}$ (grey circles, with second-order polynomial fitting line), $42 \mathrm{C}$ (black squares, with second-order polynomial fitting line), and $50^{\circ} \mathrm{C}$ (grey triangles, with exponential fitting line). Viscosity was increased at lower temperatures, with critical shear rates lower at lower temperatures.

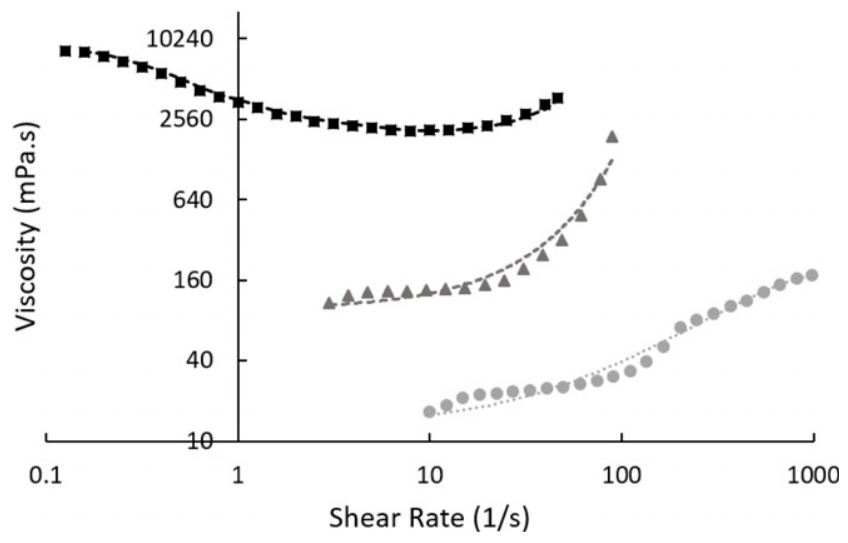

Fig. 5. Viscosity of different starches in water $(50 \% \mathrm{w} / \mathrm{w})$ as a function of shear rate. Starch was sourced from rice (black squares, with moving average fitting line), corn (grey triangles - hydroxyethyl starch, with exponential fitting line), and wheat (grey circles, with second-order polynomial fitting line). Measurements were taken at $22^{\circ} \mathrm{C}$ in a rotational rheometer. Non-Newtonian behaviour was observed in all examined mixtures - thinning with rice starch and shear thickening from all other sources.

and fructose increased the viscosity of the mixes much more than glucose, as has been reported in similar systems [1].

Biological systems are often exposed to low temperatures, and even in warm-blooded mammals' extremities can fall well below core body temperatures. Low temperatures can be induced clinically, for example cardiac and thoracic aortic surgeries are often carried out at temperatures as low at $12^{\circ} \mathrm{C}$ [6]. Viscosity is a critical factor in cryopreservation, in particular vitrification $[9,23]$. The decrease in critical shear rates seen at lower temperatures presents major advantages to their use - shear forces can be damaging to biological tissues [11,24,25], particularly after long exposure and this observation will reduce this risk in biologics as only a low shear needs to be applied before reaching critical rates. At 


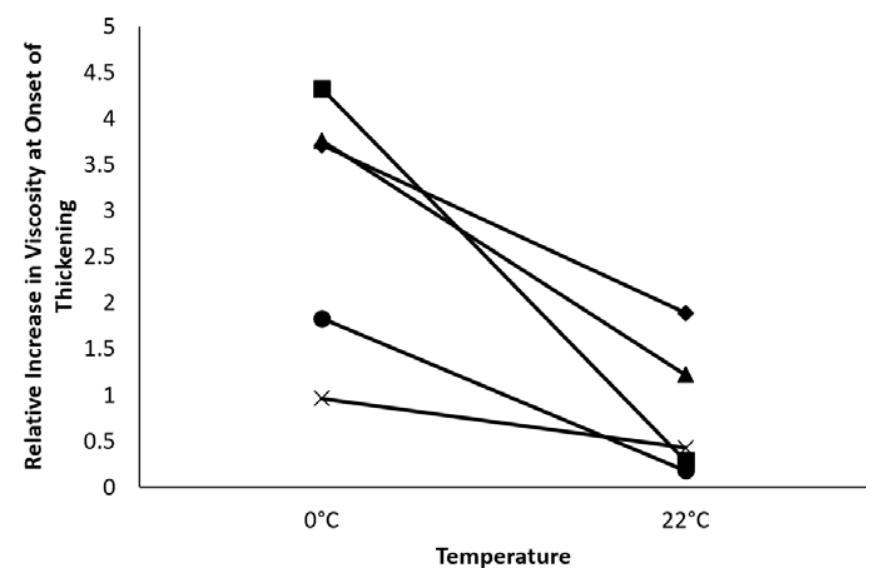

Fig. 6. Relative increase in viscosity at the onset of shear thickening (defined as from when shear-thickening commences until reaching $5 \mathrm{x}$ the pre-thickening viscosity). Reagents were measured using a concentric cylindrical rheometer at both $22^{\circ} \mathrm{C}$ and $0^{\circ} \mathrm{C}$, with a mixture of $50 \% \mathrm{HES}, 40 \%$ water, and $10 \%$ test reagent. Test Reagents were raffinose (square), sucrose (triangle), fructose (diamond), glucose (circle), and DMSO (cross).

super-critical shear rates, the macroscopic solidification of the colloid may protect biologics contained within from further damage. This temperature effect has been observed in silica suspensions as well as aqueous suspensions of polymers [26-28], and may be a consequence of slower molecular movement at lower temperatures. In this work, the critical shear rate was found to scale with a factor $0.6978 \mathrm{e}^{0.00681 \mathrm{~T}}$, where $\mathrm{T}$ is the temperature in degrees Celsius, and is above the freezing point of the solution, indicating that the relative decrease in critical shear rate is much more pronounced near the solution's freezing point. The data in this paper was carried out in a rotational rheometer. Shear-thickening behaviour was not seen in these HES mixtures using an oscillatory rheometer (data not shown), suggesting that the shear thickening may be caused by long clusters of starch particles forming a chain - these would not be expected to form in oscillatory movements. Even in approximately spherical particles, contact can be made between spheres forming these long clusters which can develop between two surfaces moving in separate directions. In such a set-up the relative incompressibility of the rigid spheres hinders the movement of the two surfaces and so the viscosity of the material apparently increases. As such a chain will only support tangential loads, varying the direction of the shear will result in chains collapsing and so shear thickening would not be seen in an oscillating set-up [15,29].

The rate of increase in viscosity while changing the shear rate around the critical rate was much more pronounced at lower temperatures - this was not expected at the outset of this study. This supports the chain hypothesis. Chains are more stable at lower temperatures (so occur at lower shear rates), as Brownian motion will take longer to break the chains at lower temperatures than higher ones, and starch particles may become more rigid, strengthening the chains in existence.

However, the full reasons for shear thickening phenomena are still not fully understood and it forms an area of active research $[2,4,5,15,30,31]$. Several hypotheses have been proposed. Hydroclusters have long been reported responsible for shear thickening, in particular during reversible continuous shear thickening. These are clusters which stick together through liquid lubrication forces [27]. However more recently friction in shear thickening has been understood necessary for the phenomenon to occur. Models have shown that perfectly smooth hard sphere systems exhibit shear thinning, and so surface topography is a key factor behind the thickening effect [27,30,32-34]. At low shear rates particles may repel each other 


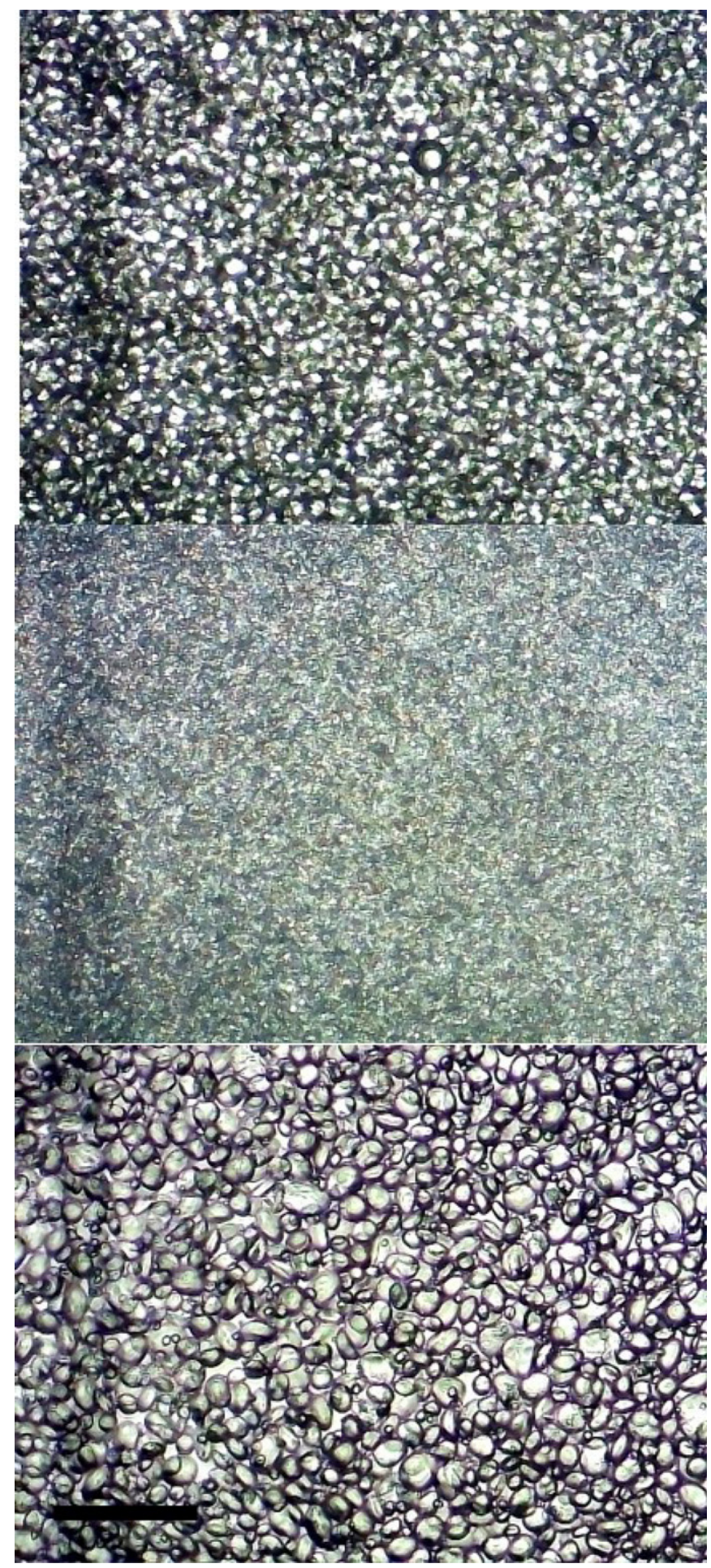

Fig. 7. Microscopy of starch from different sources. Top to bottom $-50 \%$ HES in water; $50 \%$ starch from rice in water; $50 \%$ starch from wheat in water. Scale bar is $100 \mu \mathrm{m}$. Images were taken in a static system, where no shear was applied. 
and so remain in a frictionless suspension. At higher shear rates, this repulsion is overcome and clustering can form which greatly increases particle-particle friction as jamming can occur [4,15].

The shear thickening seen with high concentrations of HES also raises the possibility that localized HES accumulation (which has been reported from blood expanders) to concentrations tested in this work may induce local shear-thickening behaviour in tissues and arteries, which may explain its clinically undesirability $[6,20-22]$. This highlights the need for an effective washing step if HES-based mixtures were to be used for cryopreservation.

It is clear from the data in Figs 5 and 7 that the source and structure of the starch is critically important to the shear response viscosity. All three sources tested have radically different physical structure and non-Newtonian behaviour. Different starch sources, while nominally very similar chemically, produce vastly different viscosity interactions. This is indicative that the mechanism behind shear thickening is very sensitive to physical parameters, perhaps related to molecular size, deformability, composition, or deviations from the spherical on the particle surfaces [35]. As can be seen from Fig. 7, while the starch particles are roughly spherical, substantial deviation in size and shape is present, a likely cause of the different shear response seen in Fig. 5. It should be noted that while starches are formed of long carbohydrates, they form discrete units in solution (Fig. 7) and when powdered - these mixtures do present a colloid as have widely been reported $[2,5,15]$. This structure was unchanged after the colloidal suspension was sheared and then relaxed (data not shown).

Starch derived from wheat exhibited shear-thinning behaviour over the range tested, which is typical of many colloids as increased shear can break-up clusters homogenising the fluid or cause particle deformation [7]. The shear thinning is not extreme (i.e. the shear stress does not reduce or plateau) so we do not suspect shear banding here. Previous work in this area has highlighted the effect that the differences between these starches have [35].

The substantial variation in shear response was not limited to particle source. This can be seen from the variations between different disaccharides in Fig. 3, where the shear-thickening behaviours of mixtures containing sucrose and lactose are markedly different despite being isomers. This indicates that both the surface friction of the particles as well as the lubrication forces of the colloid's liquid component being key parameters. Lactose is a disaccharide consisting of glucose and galactose, while sucrose consists of glucose and fructose. Switching the liquid component of the colloidal solution between solutions of these isomers causes the system to switch between a continuous shear thickening response (sucrose) to a near discontinuous shear thickening fluid (lactose). While only one gap size was examined in this study $(1.2 \mathrm{~mm})$, and larger gap sizes may result in higher critical shear rates [15]. It is possible that for this technique to be used in larger biologics (and so with a larger gap-size) lower temperatures would be required to maintain acceptable shear rates during cryopreservation.

Based on these data, we propose that in the biological systems tested, both lubrication and friction forces play a key role in the non-Newtonian behaviour observed.

\section{Conclusion}

This study establishes that the treatments used to convert corn starch to HES suitable for clinical use do not prevent shear-thickening behaviour. Adding further commonly used reagents such as sugars, DMSO, or glycerol also does not prevent this phenomenon. This would allow these fluids to be used in biological settings if required. Low temperature enhancment of shear thickening allows for their use at lower shear rates where lower temperatures are acceptable biologically. This also allows for colloids which 
display limited shear thickening at higher temperatures to be used effectively where the temperature can be lowered, or if shear thickening is undesirable, increasing the temperature could prevent the phenomenon. Large ranges in behaviour were recorded between isomers, highlighting the difficulty in definitively determining the cause of shear thickening in some colloids.

\section{References}

[1] Abu-Jdayil B, Mohameed HA, Eassa A. Rheology of wheat starch-milk-sugar systems: effect of starch concentration, sugar type and concentration, and milk fat content. Journal of Food Engineering. 2004;64:207-12.

[2] Wagner NJ, Brady JF. Shear thickening in colloidal dispersions. Physics Today. 2009;62:27-32.

[3] Wagner NJ, Wetzel ED. Advanced body armor utilizing shear thickening fluids. Google Patents. United States, 2007.

[4] Madraki Y, Hormozi S, Ovarlez G, Guazzelli E, Pouliquen O. Enhancing shear thickening. Physical Review Fluids. 2017;2:033301.

[5] Barnes H. Shear-thickening ("Dilatancy") in suspensions of nonaggregating solid particles dispersed in Newtonian liquids. Journal of Rheology (1978-present). 1989;33:329-66.

[6] Eckmann DM, Bowers S, Stecker M, Cheung AT. Hematocrit, volume expander, temperature, and shear rate effects on blood viscosity. Anesthesia \& Analgesia. 2000;91:539-45.

[7] Pries A, Secomb T. Rheology of the microcirculation. Clinical hemorheology and microcirculation. 2003;29:143-48.

[8] Fuller BJ, Lane N, Benson EE. Life in the Frozen State. Boca Raton, FL: CRC Press; 2004. p. 672.

[9] Fahy GM, Wowk B, Wu J, Phan J, Rasch C, Chang A, Zendejas E. Cryopreservation of organs by vitrification: perspectives and recent advances. Cryobiology. 2004;48:157-78.

[10] Meryman HT. Cryopreservation of living cells: Principles and practice. Transfusion. 2007;47:935-945.

[11] Lagerstedt $\AA$, Enfält L, Johansson L, Lundström K. Effect of freezing on sensory quality, shear force and water loss in beef M. longissimus dorsi. Meat Science. 2008;80:457-61.

[12] Giwa S, Lewis JK, Alvarez L, Langer R, Roth AE, Church GM, Markmann JF, Sachs DH, Chandraker A, Wertheim JA. The promise of organ and tissue preservation to transform medicine. Nature Biotechnology. 2017;35:530.

[13] Fahy GM, Wowk B, Wu J. Cryopreservation of complex systems: the missing link in the regenerative medicine supply chain. Rejuvenation Research. 2006;9:279-91.

[14] Kilbride P, Lamb S, Gibbons S, Bundy J, Erro E, Selden C, Fuller B, Morris J. Cryopreservation and re-culture of a 2.3 litre biomass for use in a bioartificial liver device. PloS One. 2017;12:e183385.

[15] Fall A, Huang N, Bertrand F, Ovarlez G, Bonn D. Shear thickening of cornstarch suspensions as a reentrant jamming transition. Physical Review Letters. 2008;100:018301.

[16] Huang X, Qian W, El-Sayed IH, El-Sayed MA. The potential use of the enhanced nonlinear properties of gold nanospheres in photothermal cancer therapy. Lasers in surgery and medicine. 2007;39:747-53.

[17] Mahon E, Salvati A, Bombelli FB, Lynch I, Dawson KA. Designing the nanoparticle-biomolecule interface for "targeting and therapeutic delivery". Journal of Controlled Release. 2012;161:164-74.

[18] Elliott GD, Wang S, Fuller BJ. Cryoprotectants: A review of the actions and applications of cryoprotective solutes that modulate cell recovery from ultra-low temperatures. Cryobiology. 2017;76:74-91.

[19] Telis V, Telis-Romero J, Mazzotti H, Gabas A. Viscosity of aqueous carbohydrate solutions at different temperatures and concentrations. International Journal of Food Properties. 2007;10:185-95.

[20] Antonelli M, Sandroni C. Hydroxyethyl starch for intravenous volume replacement: more harm than benefit. Jama. 2013;309:723-24.

[21] Wiedermann CJ, Joannidis M. Accumulation of hydroxyethyl starch in human and animal tissues: a systematic review. Intensive Care Medicine. 2014;40:160-70.

[22] Zarychanski R, Abou-Setta AM, Turgeon AF, Houston BL, McIntyre L, Marshall JC, Fergusson DA. Association of hydroxyethyl starch administration with mortality and acute kidney injury in critically ill patients requiring volume resuscitation: a systematic review and meta-analysis. Jama. 2013;309:678-88.

[23] Kilbride P, Morris G. Viscosities encountered during the cryopreservation of dimethyl sulphoxide systems. Cryobiology. 2017;76:92-7.

[24] Vanbavel E. Effects of shear stress on endothelial cells: possible relevance for ultrasound applications. Progress in Biophysics and Molecular Biology. 2007;93:374-83. 
[25] Nobili M, Sheriff J, Morbiducci U, Redaelli A, Bluestein D. Platelet activation due to hemodynamic shear stresses: Damage accumulation model and comparison to in vitro measurements. ASAIO journal (American Society for Artificial Internal Organs: 1992). 2008;54:64.

[26] Warren J, Offenberger S, Toghiani H, Pittman Jr CU, Lacy TE, Kundu S. Effect of temperature on the shear-thickening behavior of Fumed silica suspensions. ACS Applied Materials \& Interfaces. 2015;7:18650-61.

[27] Lin NY, Guy BM, Hermes M, Ness C, Sun J, Poon WC, Cohen I. Hydrodynamic and contact contributions to continuous shear thickening in colloidal suspensions. Physical Review Letters. 2015;115:228304.

[28] Ma SX, Cooper SL. Shear thickening in aqueous solutions of hydrocarbon end-capped poly (ethylene oxide). Macromolecules. 2001;34:3294-301.

[29] Cates M, Wittmer J, Bouchaud J-P, Claudin P. Jamming, force chains, and fragile matter. Physical Review Letters. 1998;81:1841.

[30] Seto R, Mari R, Morris JF, Denn MM. Discontinuous shear thickening of frictional hard-sphere suspensions. Physical Review Letters. 2013;111:218301.

[31] Fernandez N, Mani R, Rinaldi D, Kadau D, Mosquet M, Lombois-Burger H, Cayer-Barrioz J, Herrmann HJ, Spencer ND, Isa L. Microscopic mechanism for shear thickening of non-Brownian suspensions. Physical Review Letters. 2013;111:108301.

[32] Heussinger C. Shear thickening in granular suspensions: Interparticle friction and dynamically correlated clusters. Physical Review E. 2013;88:050201.

[33] Guy B, Hermes M, Poon W. Towards a unified description of the rheology of hard-particle suspensions. Physical Review Letters. 2015;115:088304.

[34] Gallier S, Lemaire E, Peters F, Lobry L. Rheology of sheared suspensions of rough frictional particles. Journal of Fluid Mechanics. 2014;757:514-49.

[35] Singh N, Singh J, Kaur L, Sodhi NS, Gill BS. Morphological, thermal and rheological properties of starches from different botanical sources. Food Chemistry. 2003;81:219-31. 